\title{
Perturbative saturation and the soft Pomeron
}

\author{
A. Kovner \\ Department of Mathematics and Statistics, University of Plymouth, 2 Kirkby Place, Plymouth, PL4 8AA, United Kingdom \\ and Department of Physics, University of Connecticut, Storrs, Connecticut 06269 \\ U. A. Wiedemann \\ CERN, Theory Division, CH-1211 Geneva 23, Switzerland
}

(Received 24 April 2002; published 28 August 2002)

\begin{abstract}
We show that perturbation theory provides two distinct mechanisms for the powerlike growth of hadronic cross sections at high energy. One, the leading Balitskiü-Fadin-Kuraev-Lipatov (BFKL) effect, is due to the growth of the parton density, and is characterized by the leading BFKL exponent $\omega$. The other mechanism is due to the infrared diffusion, or the long range nature of the Coulomb field of perturbatively massless gluons. When perturbative saturation effects are taken into account, the first mechanism is rendered ineffective but the second one persists. We suggest that these two distinct mechanisms are responsible for the appearance of two Pomerons. The density growth effects are responsible for the hard Pomeron and manifest themselves in small systems (e.g. $\gamma^{*}$ or small size fluctuations in the proton wave function) where saturation effects are not important. The soft Pomeron is the manifestation of the exponential growth of the black saturated regions which appear in typical hadronic systems. We point out that the nonlinear generalization of the BFKL equation which takes into account wave function saturation effects ("Pomeron loops") provides a well defined perturbative framework for the calculation of the soft Pomeron intercept. The conjecture of a perturbative soft Pomeron is consistent with picturing the proton as a loosely bound system of several small black regions corresponding e.g. to constituent quarks of size about $0.3 \mathrm{fm}$. Phenomenological implications of this picture are compatible with the main qualitative features of data on $p-p$ scattering.
\end{abstract}

DOI: 10.1103/PhysRevD.66.034031

PACS number(s): 12.38.Bx, 13.60.Hb

\section{INTRODUCTION}

It has been long recognized that at asymptotically high energies hadronic cross sections are dominated by soft nonperturbative physics. In particular the validity of the Froissart bound for the total cross section requires a mass gap in the spectrum. The corresponding generation of the pion mass in QCD, or the generation of the glueball mass in pure gluodynamics, is a bona fide nonperturbative effect. However, even though the asymptotics is expected to be nonperturbative, perturbative dynamics may well play an important role in the preasymptotic regime.

Indeed, cross sections for small objects, like highly virtual photon $\gamma^{*}$, or heavy quarkonium ("onium") are perturbative up to very high energies, as long as the size of the system remains small. The main perturbative mechanism that drives the growth of cross sections with energy is the Balitski1Fadin-Kuraev-Lipatov (BFKL) evolution [1]. It predicts that cross sections grow exponentially, $\sigma \propto s^{\omega}$, where $\omega$ $=4 \ln 2 N_{c}\left(\alpha_{s} / \pi\right)$ to leading order in $\alpha_{s}$. This behavior is nonunitary, as it violates the Froissart bound $\sigma \leqslant\left(\pi / \mathrm{m}^{2}\right) t^{2}$. Here $t$ is the rapidity, $t=\ln \left(s / m^{2}\right)$, and $m$ denotes the mass of the lightest particle in the theory. The main reason for this lack of unitarity is the growth of the partonic density in the hadronic system. As the partonic density reaches a critical value of order $1 / \alpha_{s}$, the BFKL approach ceases to be valid, and one must take into account finite density effects.

This has been recognized and forcefully advocated in the pioneering works [2] and later in [3]. A more complete approach to QCD evolution at finite density has been developed more recently in [4]. These papers treat the QCD evo- lution to the leading logarithmic approximation, but to all orders in the gluon density. The resulting system of evolution equations is a set of functional equations, and its study so far has not been feasible beyond the double logarithmic approximation.

There exists however a regime in which the general nonlinear evolution simplifies. This happens when a small object (such as a highly virtual photon) scatters on a large target (such as a large nucleus). In this case the leading nonlinear corrections are due to the fact that the projectile wave function at high energy has a large multigluon component. As soon as the number of gluons in these multigluon states becomes large, one has to account for the possibility that more than one gluon scatters, even if the gluonic density may still be small. When the target is large, so that the scattering probability of a gluon is parametrically larger than $\alpha_{s}$ [for a large nucleus of atomic number $A$, it is $\left.O\left(A^{1 / 3} \alpha_{s}\right)\right]$, these corrections become important earlier than those due to high density effects.

The system of evolution equations which takes into account these multiple scattering effects has been derived first in [5]. In [6] its large $N_{c}$ limit was derived independently in the dipole picture of [7]. The advantage of the large $N_{c}$ limit is that the (otherwise infinite) hierarchy of evolution equations closes and becomes a single equation for the gluon density (or dipole scattering probability). We will refer to this nonlinear equation as the Balitsky-Kovchegov (BK) equation. The relation between the equations of [4] and [5] has been discussed in [8], where it was shown that the latter is the limit of the former when the induced field density 
is small. This result was later rederived by similar methods in [9]. ${ }^{1}$

The range of validity of the $\mathrm{BK}$ equation is wider than that of the BFKL evolution in the sense that it can be applied to scattering on large targets. If the target is large so that the scattering probability of a given probe on it is of order unity, the BFKL evolution violates unitarity very quickly, at rapidities of order $t_{B F K L} \propto 1 / \alpha_{s} N_{c}$. This violation of unitarity stems from the scattering probability rising above one locally in impact parameter space. In contrast with BFKL, the BK evolution ensures that the scattering probability at a given impact parameter is always below unity. Nevertheless, as we will discuss in the following, for scattering of a (small) projectile of transverse size $x_{0}$ on a (large) target of size $R_{0}$, the BK evolution violates unitarity for rapidities $t>t_{B K}$ $\propto 1 / \alpha_{s} N_{c} \ln \left(R_{0} / x_{0}\right)$. The reason for this violation is that the value of the maximal impact parameter that contributes to scattering grows exponentially with rapidity. Thus even though the scattering probabilities remain unitary, the total cross section grows exponentially, $\sigma \propto \exp \{\epsilon t\}$, and violates the Froissart bound. This is the main result of [10]. We can give a crude estimate of the exponent $\epsilon$, but we are not able to perform a reliable analytical calculation at this point. However the numerical results of [11] give $\epsilon=0.75 \omega$, which is indeed compatible with our rough estimate. Thus the BK exponent is smaller than the BFKL one, but not very significantly.

The reason why at high enough energy the BK evolution ceases to be valid is that the projectile wave function becomes dense, and further evolution is affected by the high density effects in the projectile wave function. The onset of these corrections is at $t \propto 1 / \alpha_{s} N_{c} \ln \left(1 / \alpha_{s}\right)$. As discussed below, the gluon density in the center of the projectile is large at energies at which large values of the impact parameter become dominant. Thus, finite density corrections in the central region are important. Their main direct effect is to slow down the growth of density in the central region, but this must also have a large effect on the growth of the scattering probability at large impact parameters. As we will discuss below, this growth at large impact parameter is due to the long range Coulomb (or Weizsäcker-Williams) fields originating from the fluctuation of the color charge in the central "black" region. The finite density effects inevitably reduce the magnitude of these fluctuations and therefore also the Coulomb fields which feed the periphery. We expect therefore that these corrections are likely to reduce the exponential $\epsilon$ significantly. The proper calculation of the exponential $\epsilon$ must involve the analysis of the full nonlinear equation, including the wave function saturation effects [4].

\footnotetext{
${ }^{1}$ We note that although it is claimed verbally in [9] that the equations of [4] should be equivalent to those of [5] not only for small induced fields, but also in general, the actual mathematical analysis of [9] does not justify this claim. The mathematical analysis of [9] (up to notational differences) is equivalent to that of [8], where the origin of the differences between the results of [5] and [4] has been discussed.
}

One aim of the present paper is to furnish more details to the derivations of [10]. This is done in Secs. III and IV, after the short recourse to the BFKL evolution in Sec. II. The other aim is to reflect on possible implications of these two distinct perturbative mechanisms for the powerlike growth of the total cross section. The first mechanism-the growth of partonic density - is naturally associated with small systems. As long as the size of the system is small and also the number of partons not very large, one expects that the growth of density is indeed the leading mechanism. This suggests that the BFKL exponent (possibly modified by higher order corrections) dominates the scattering cross section of small systems. However, if a typical hadron is a dense partonic system rather than a dilute one, then the density growth mechanism is rendered irrelevant by saturation effects. It is only the powerlike growth due to the perturbative expansion in the transverse plane which remains effective in this case. One is thus led naturally to conjecture, that it is this latter mechanism that is responsible for the experimentally found "soft Pomeron" behavior of hadronic cross sections.

Associating the soft Pomeron with a perturbative phenomenon is certainly unconventional. All models of the soft Pomeron that we are aware of, try to explain the power growth of cross sections by a nonperturbative mechanism $[12,13]$. On the other hand, this power growth of the cross section implies by the extension of Heisenberg's argument [14] the powerlike distribution of "matter" in impact parameter space. Perturbation theory naturally provides such a mechanism, since it operates with massless gluons and thus long range Coulomb fields. Thus it could well be that the role of nonperturbative physics is limited to taming the powerlike perturbative growth in asymptotia, rather than to provide an additional mechanism for a powerlike growth in the preasymptotic regime. $^{2}$

For this scenario to be viable, the basic saturated ("black") building blocks of hadrons must be themselves small in size, so that the perturbative Coulomblike structure of the gluon fields is still relevant at these distances. It has been pointed out repeatedly in the literature that the distance scale associated with the constituent quarks is much smaller than the scale of confining physics. Indeed, the models $[12,13]$ deal with scales of order .3 fermi. The picture we suggest therefore, is that of a proton containing three small "black" constituent quarks with the size about .3 fermi in the proton rest frame. As the proton is boosted, the radius of constituent quarks grows with half the soft Pomeron power, $r \propto s^{.04}$. The mechanism of this growth is perturbative and the power itself should be calculable from the full nonlinear QCD evolution equation [4]. Section V of the present paper is devoted to a more detailed discussion of this scenario.

\footnotetext{
${ }^{2}$ We note that the models of $[12,13]$, when translated into our language try to provide an alternative nonperturbative mechanism for the growth of the density rather than for the growth in impact parameter space. Since the exponent governing this growth is supposed to be much smaller than the perturbative one, it is hard to see how this sort of mechanism can survive as the leading one when juxtaposed with BFKL.
} 


\section{THE TWO EXPONENTS OF THE BFKL EQUATION}

To illustrate the perturbative features of high energy reactions, we choose the example of deeply inelastic scattering (DIS). The subprocess relevant to strong interaction physics is the scattering of the photon of virtuality $Q^{2}$ on a proton. In the parton model, the photon counts the number of charged partons in the proton. The scattering cross section is then

$$
\sigma_{\mathrm{DIS}}(Q, x)=\frac{\alpha_{e m}}{Q^{2}} \sum_{i} e_{i}^{2} N_{i}(Q, x),
$$

where the first factor is the parton level cross section while $N_{i}$ is the number of partons of a given species in the proton. The number of partons depends on both the resolution scale $Q^{2}$ and the energy at which the proton wave function is probed, $x=\left[Q^{2} /\left(Q^{2}+W^{2}\right)\right]$. Here $W$ denotes the center of mass energy of the $\gamma^{*} p$ system. One can define the phase space density of partons, $\phi(k)$, in terms of which

$$
N\left(Q^{2}, x\right)=\int_{S} d^{2} b \int_{k^{2} \leqslant Q^{2}} d^{2} k \phi(b, k, x),
$$

where $k$ is the intrinsic transverse momentum of the parton, and $b$ is the impact parameter at which the parton is found in the transverse plane.

Although the photon directly counts the number of charged partons, i.e. quarks, at low $x$ the proton wave function is dominated by gluons. The number of quarks is then directly determined by the gluon content of the wave function. In the following we will therefore concentrate on the gluons only.

At low $x$, in the leading logarithmic approximation, the gluon density $\phi$ is determined by the asymptotic solution of the BFKL equation [1]. According to this solution, the distribution of gluons is

$$
\phi\left(b, k, x, k_{0}\right) \propto \exp \left\{\omega t-\frac{\ln ^{2} b^{2} k k_{0}}{a^{2} t}\right\} .
$$

Here $t=\ln 1 / x$ is the rapidity, $\omega=4 \ln 2 N_{c} \alpha_{s} / \pi$ and $a^{2}$ $=14 \zeta(3) N_{c} \alpha_{s} / \pi$ with $\zeta(n)$ being the Riemann zetafunction, $\zeta(3)=1.202 \ldots$. This distribution depends also on $k_{0}$ which characterizes the initial transverse momentum of the gluon which gave rise to $\phi$ through the evolution to high $t$. Formula (3) is valid for impact parameters which are not too large, namely

$$
0 \leqslant \ln b^{2} k k_{0} \ll \alpha_{s} t .
$$

The striking feature of Eq. (3) is that even if at low rapidity $t_{0}$ one starts with a single gluon, after evolution to high enough $t$, the density of gluons at small impact parameters becomes exponentially large. Within the diffusion radius $\ln b \propto a t^{1 / 2}$, the overall scale of density is determined by the exponential factor $\exp \{\omega t\}$. Thus using Eq. (3) in the gluonic analog of Eq. (1) gives the total cross section which grows exponentially with rapidity.
The high density at central impact parameters is not the only source of growth in Eq. (3). The gluon density does decrease towards the peripheral impact parameters, but it does so rather slowly. For a given transverse momentum $k$, the density stays finite up to impact parameters of order $\ln \left(b^{2} k k_{0}\right) \propto \alpha_{s} t$. One cannot establish the exact proportionality coefficient from Eq. (3), since the validity of this equation does not extend to such large impact parameters. However impact parameters of order $\ln \left(b^{2} k k_{0}\right)=\nu \alpha_{s} t$ with very small $\nu$ are still covered by Eq. (3). For such impact parameters the density is still exponentially large, although parametrically smaller than inside the "diffusion radius." These peripheral impact parameters do not contribute to the leading BFKL exponential. When the density is integrated over the impact parameter plane to calculate the total number of gluons, one obtains the leading BFKL result $N(t)=\int d^{2} b \phi(b, t)$ $=a \exp \omega t$ which is dominated by the impact parameters within the diffusion radius. However the contribution of the peripheral region is by itself also exponentially increasing. Were we to exclude the central impact parameters from the integration region, we would still get an exponentially large contribution of the form $\exp \left\{\epsilon \alpha_{s} t\right\}$. The exponent here is smaller than the leading BFKL one, and thus gives a negligible contribution to the total cross section within the BFKL framework. However, this exponent is nevertheless present, and its physical origin is quite distinct from the exponential growth of partonic density.

The exponential growth of the cross section violates the Froissart bound which requires the total cross section to grow not faster than the second power of the logarithm of energy

$$
\sigma_{\text {total }} \leqslant \frac{\pi}{m_{\pi}^{2}} \ln ^{2} \frac{s}{m_{\pi}^{2}}
$$

The leading exponential growth of the BFKL cross section is of course unphysical at high enough energies. Taken at its face value Eq. (1) in conjunction with Eq. (3) would mean that the probability for scattering of a strongly interacting probe at fixed impact parameter grows without bound at large energy. This is inconsistent with the fact that the probability cannot exceed 1 , and violates unitarity of the scattering probability at fixed impact parameter. The reason is that a strongly interacting probe has finite probability to interact with more than one gluon at fixed $b$ when the gluon density at fixed impact parameter and fixed resolution $Q^{2}$ exceeds $1 / \alpha_{s}$. Thus the cross section is not proportional to the number of gluons anymore. Multiple scattering effects must be properly taken into account in order to relate the gluon density with the scattering probability.

A nonlinear QCD evolution equation that takes into account these multiple scattering effects has been derived by Balitsky [5]. Its large $N_{c}$ version was obtained by Kovchegov [6] using Mueller's dipole model approach. In the next two sections we will discuss how far this perturbative resummation goes beyond the simple BFKL framework towards restoring the unitarity of the hadronic cross sections.

We start our discussion with considering the evolution in the target rest frame. 


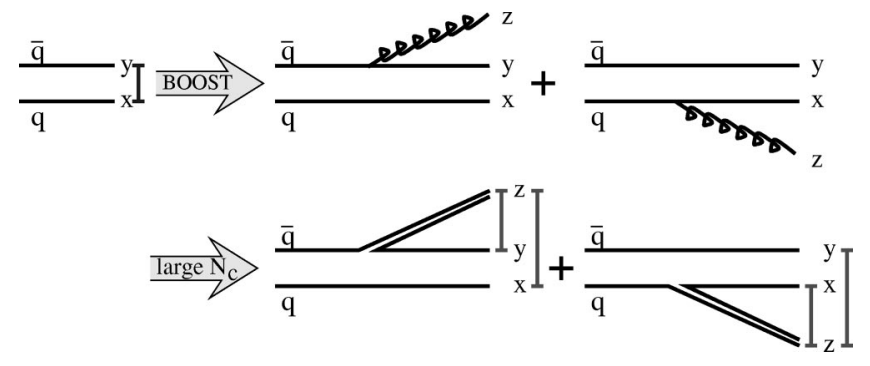

FIG. 1. Boosting the $q \bar{q}$-dipole $(x, y)$ generates the higher Fock component $|q(x) \bar{q}(y) g(z)\rangle$ with a gluon at transverse position $z$. In the large $N_{c}$-limit, this corresponds to the generation of dipoles $(x, z)$ and $(y, z)$.

\section{THE BK EVOLUTION IN THE TARGET REST FRAME}

The essence of the BK evolution is the following. Suppose at some initial rapidity $t_{0}$ we are interested in the scattering of a probe consisting of a $\bar{q} q$ dipole on a large hadronic target, for example a heavy nucleus. The scattering probability of a dipole with legs at transverse coordinates $x$ and $y$ on a hadronic target is $N(x, y)$. Increasing the center of mass energy (or rapidity) amounts to boosting the dipole to rapidity $t=t_{0}+\delta t$. Under boost, the longitudinal Coulomb field associated with the dipole acquires a transverse part, i.e., the dipole of transverse size $x-y$ generates an extra gluonic component whose density is given by the equivalent gluon content of the Weizsäcker-Williams field of the dipole, see Fig. 1:

$$
|q(x) \bar{q}(y)\rangle \rightarrow A|q(x) \bar{q}(y) g(z)\rangle
$$

with

$$
A^{2}=\frac{\alpha_{s} N_{c}}{2 \pi^{2}} \delta t \frac{(x-y)^{2}}{(x-z)^{2}(x-y)^{2}} .
$$

In the large $N_{c}$ limit, the gluon is equivalent to a quarkantiquark pair. This and the global color conservation of the QCD evolution implies that the singlet $\bar{q} q g$ state is equivalent to two dipoles with coordinates $x, z$ and $y, z$ respectively, see Fig. 1. In the leading large $N_{c}$ approximation, the dipoles scatter independently of each other. Thus the probability for the scattering of the pair of dipoles is

$$
N(x, z ; y, z)=N(x, z)+N(y, z)-N(x, z) N(y, z) \text {. }
$$

The last negative term, $N(x, z) N(y, z)$, is the probability that both dipoles scatter in the same collision. Such double scattering events should be counted once and not twice in the total cross section, and the last term in Eq. (8) corrects the overcounting of $N(x, z)+N(y, z)$.

This leads to the following nonlinear evolution equation for the dipole scattering probability:

$$
\begin{aligned}
\frac{d}{d t} N(x, y)= & \frac{\alpha_{s} N_{c}}{2 \pi^{2}} \int d^{2} z \frac{(x-y)^{2}}{(x-z)^{2}(y-z)^{2}}[N(x, z)+N(y, z) \\
& -N(x, z) N(z, y)-N(x, y)]
\end{aligned}
$$

The first three terms in Eq. (9) are just the ones discussed above, while the last term is the "virtual" correction which ensures that the dipole wave function stays normalized throughout the evolution.

The following two properties of the BK equation Eq. (9) are important for our discussion. First, as stressed above, it takes into account multiple scattering corrections. Second, within this approach the projectile wave function evolves according to the linear evolution of the dipole model. Interactions between the dipoles in the projectile wave function are not taken into account. This is seen explicitly e.g. from the original derivation of [6] where the density of dipoles in the projectile wave function $n_{1}(x, y)$ satisfies the linear BFKL evolution equation,

$$
\begin{aligned}
\frac{d}{d t} n_{1}(x, y)= & \frac{\alpha_{s} N_{c}}{2 \pi^{2}} \int d^{2} z\left(\frac{1}{(y-z)^{2}} n_{1}(x, z)\right. \\
& +\frac{1}{(x-z)^{2}} n_{1}(y, z) \\
& \left.-\frac{(x-y)^{2}}{(x-z)^{2}(y-z)^{2}} n_{1}(x, y)\right) .
\end{aligned}
$$

The nonlinearity in the BK evolution equation comes not from the nonlinearities in the evolution of the projectile wave function, but from the nonlinearity in the relation between the dipole density and the scattering probability. This is again given explicitly in [6]. A single dipole $\left(x_{0}, y_{0}\right)$ at initial rapidity $t_{0}$ develops at a greater rapidity $t$ into a wave function characterized by the $m$-dipole densities, $n_{m}\left(x_{0}, y_{0}, t_{0} \mid x_{1}, y_{1} ; \ldots ; x_{m}, y_{m}, t\right)$. If the single dipole scattering probability at $t_{0}$ is $\gamma(x, y)$, the total scattering probability is given by [6]

$$
\begin{aligned}
N\left(x_{0}, y_{0}, t\right)= & \sum_{m}\left(\prod_{i=1}^{m} \gamma\left(x_{i}, y_{i}\right)\right) \\
& \times n_{m}\left(x_{0}, y_{0}, t_{0} \mid x_{1}, y_{1} ; \ldots ; x_{m}, y_{m}\right) .
\end{aligned}
$$

The scattering probability $\gamma$ depends on the target, but not on the rapidity.

The account of multiple scatterings in the BK resummation eliminates the leading mechanism that renders the BFKL evolution nonunitary. Since the scattering probability in the BK evolution is no longer proportional to the gluon density, the scattering probability at any impact parameter does not exceed unity. This is obvious from Eq. (9). When the scattering probability $N(x, y)$ reaches unity at all impact parameters, the right hand side of the evolution equation vanishes, and the probability stops growing.

A number of numerical [15-17] as well as analytical $[18,19]$ studies of Eq. (9) have been performed, and they all lead to the following consistent picture: Suppose one starts the evolution from the initial condition of small target fields [or $N(x, y) \ll 1$ for all $x, y$ ]. Then initially the evolution follows the BFKL equation, since the nonlinear term in Eq. (9) is negligible. As the scattering probability approaches unity, the nonlinear term kicks in and eventually the growth stops 
as the right-hand side (RHS) of Eq. (9) vanishes for $N(x, y)=1$. The larger dipoles [large $\left.(x-y)^{2}\right]$ saturate earlier, with the smaller dipoles following at later "time" $t$. The following simple parametrization [20] of the scattering probability gives an adequate description of the evolution:

$$
N(x, y)=1-\exp \left\{-(x-y)^{2} Q_{s}^{2}(t)\right\}
$$

with the saturation momentum $Q_{s}(t)$ a growing function of rapidity. Thus at any given value of rapidity, all pairs of size greater than $Q_{s}^{-1}(t)$ are saturated.

The exact dependence of $Q_{s}$ on rapidity is not known, but both the numerical results $[15,17]$ and simple theoretical estimates $[21,19]$ are consistent with the exponential growth of the form

$$
Q_{s}(t)=\Lambda \exp \left\{\alpha_{s} \lambda t\right\}
$$

with $\lambda$ of order unity. This physical picture was anticipated several years ago in [21].

Does saturation of the scattering probability locally in impact parameter plane necessarily imply that the total cross section unitarizes and satisfies the Froissart bound? The answer clearly is negative. The Froissart bound states that the inelastic cross section for the scattering of a hadron (dipole) on a hadronic target cannot grow faster than the square of rapidity

$$
\sigma<\frac{\pi}{m^{2}} t^{2}
$$

where $m$ is the mass of the lightest hadronic excitation. To calculate the inelastic cross section one has to integrate the scattering probability over the impact parameter. Thus, in the saturation regime

$$
\sigma=\pi R^{2}(t),
$$

where $R(t)$ is the size of the region in the transverse plane in which the scattering probability for hadronic size "dipoles" is unity. This radius itself depends on $t$. To satisfy the Froissart bound the radius $R(t)$ should grow at most linearly with $t$. The question of unitarity is therefore the question about the rate of growth of the "black" region, and thus is completely separate from the question of saturation of the scattering probability at fixed impact parameter.

As an aside we note that for the deeply inelastic scattering the unitarity bound is somewhat different [22]. In this case the projectile is a virtual photon. It does not have a fixed hadronic size, but rather is characterized by a distribution of dipole sizes. The perturbative wave function of the virtual photon is well known. An interesting property of this wave function is that for a transverse photon it has a long tail on the small dipole side $\left(r^{2} \ll Q^{-2}\right)$

$$
\Phi^{2}(r) \propto \alpha_{e m} \frac{1}{r^{2}} .
$$

In such a projectile not all dipoles saturate at the same energy. The scattering probability thus is given by the integral over the dipole sizes. At every rapidity the main contribution comes from the dipoles which are saturated, that is those with sizes above $Q_{s}^{-1}(t)$. The scattering probability for a virtual photon at high energy (by high we mean here such that $Q_{s} \gg Q$ ), is given by

$$
N\left(\gamma^{*}\right)=\int_{r^{2}<Q_{s}^{-2}} d^{2} r \Phi^{2}(r) \propto \alpha_{e m} \ln Q_{s} / Q .
$$

With the exponential dependence of $Q_{s}$ on rapidity this translates into

$$
N\left(\gamma^{*}\right) \propto \alpha_{e m} \alpha_{s} \ln s / s_{0}
$$

and therefore

$$
\sigma_{\mathrm{DIS}} \propto \alpha_{e m} \alpha_{s} \pi R^{2}(t) t
$$

Thus the DIS cross section has an extra power of $t$ relative to the cross section of a purely hadronic process. ${ }^{3}$

This extra power of $t$ is consistent with the numerical results of [17]. The basic question of unitarization however remains the same: what is the dependence of $R$ on rapidity ?

While there is no doubt that QCD is a unitary theory, and therefore indeed $R(t) \propto t$, there is no guarantee that the nonlinear BK Eqs. (32),(9) preserve this property. In fact simple considerations indicate the opposite. This is especially clear from Kovchegov's derivation [6] (see also [23]) where the density of dipoles in the projectile wave function is explicitly determined by the BFKL equation. Saturation is the result of the multiple scattering of the dense dipole system, rather than the slowdown in the growth of the dipole density. Since the transverse size of a system in BFKL evolution grows exponentially with rapidity, there is little doubt that the BK evolution violates unitarity of the total cross section.

We now present a simple calculation that establishes this point: Consider the BK evolution as the evolution of the projectile $[6,23]$ (see Fig. 2). Suppose at the initial energy the projectile is a color dipole of size $x_{0}$. It scatters on a hadronic target of size $R_{0}$. As the energy is increased, the projectile wave function evolves according to the BFKL equation. At rapidity $t$ the density of dipoles of size $x$ at transverse distance $r$ from the original dipole is given by the BFKL expression (see for example [24]):

\footnotetext{
${ }^{3}$ The situation is very different for a vector particle which directly strongly couples to quarks. The ultraviolet structure of such a particle is affected by the running of the strong coupling constant. The extra $\alpha_{s}\left(r^{-1}\right)$ which replaces $\alpha_{e m}$ in Eq. (17) introduces a logarithmically decreasing factor which suppresses the contribution of very small pairs to the total cross section. For such a particle therefore the cross section satisfies the standard Froissart bound. Although this is entirely academic, we note that our expression for the DIS cross section is valid only at energies below $s_{c}$ such that $\alpha_{e m} \alpha_{s} \ln s_{c} / s_{0}<1$. Above this energy higher order electromagnetic corrections must become important so that $N\left(\gamma^{*}\right)$ also saturates.
} 


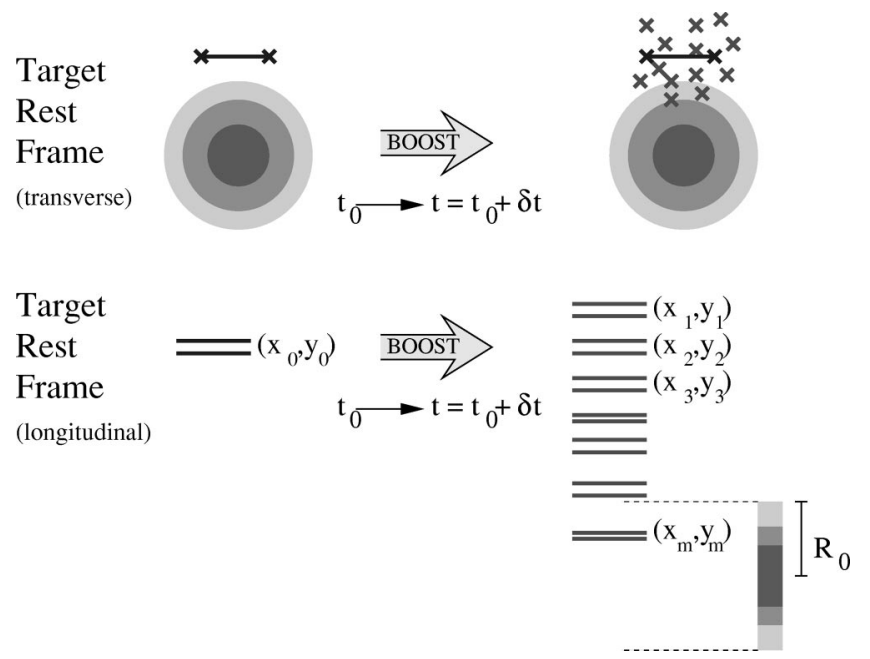

FIG. 2. The BK evolution in the target rest frame. Under boost, the initial dipole $\left(x_{0}, y_{0}\right)$ evolves into a wave function containing $m$-dipole configurations with density determined by the BFKL expression (20). The spread of these configurations in impact parameter space leads to a finite interaction probability even if the initial dipole $\left(x_{0}, y_{0}\right)$ was at very large impact parameter.

$$
n\left(x_{0}, x, b, t\right)=\frac{32}{x^{2}} \frac{\ln \frac{16 b^{2}}{x_{0} x}}{\left(\pi a^{2} t\right)^{3 / 2}} \exp \left\{\omega t-\ln \frac{16 b^{2}}{x_{0} x}-\frac{\ln ^{2} \frac{16 b^{2}}{x_{0} x}}{a^{2} t}\right\}
$$

with $\omega=4 \ln 2 N_{c} \alpha_{s} / \pi$ and $a^{2}=14 \zeta(3) N_{c} \alpha_{s} / \pi$ and $\zeta(n)$ being the Riemann zeta-function.

Once the density of dipoles at some impact parameter $b$ becomes larger than some fixed critical number, the scattering probability at this impact parameter saturates. The exact value of this number depends on the target, but importantly it does not depend on rapidity. Thus the total cross section is given by the square of the largest impact parameter at which the dipole density in the projectile wave function is of order unity. In order to estimate this directly from Eq. (20), we choose the dipole size $x$ in Eq. (20) as $x=Q_{s}^{-1}\left(t_{0}\right)$. Recall that according to Eq. (12), the dipole of this size scatters with probability 1 , if it hits inside the radius of the target $R_{0}$ (in this view of the evolution only the projectile wave function depends on energy, while the properties of the target at $t$ are the same as at $\left.t_{0}\right)$. Thus if at some impact parameter $R(t)$ the density of dipoles of size $Q_{s}^{-1}\left(t_{0}\right)$ is unity, the scattering probability at this impact parameter is unity as well; see Fig. 3. Requiring the exponential in Eq. (20) to vanish we obtain ${ }^{4}$

\footnotetext{
${ }^{4}$ More accurately one should require that the number of dipoles of size $Q_{s}^{-1}\left(t_{0}\right)$ in the area of the overlap with the target $\left(\pi R_{0}^{2}\right)$ is at least one. This refinement however only modifies the preexponential factor in Eq. (21), the calculation of which is in any case beyond the scope of this paper. To determine this preexponential factor one would have to treat more carefully the preexponential factors in Eq. (20). Equation (21) is thus valid only with exponential accuracy.
}

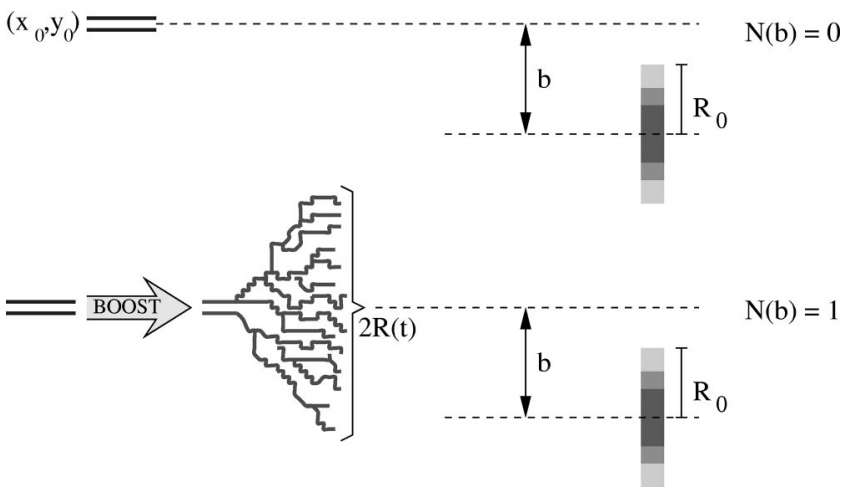

FIG. 3. Under boost, the dipole density of the BK equation evolves according to Eq. (20). If the density of dipoles of critical size $Q_{s}^{-1}\left(t_{0}\right)$ is unity at some impact parameter $b$, then the scattering probability $N(b)$ is unity as well.

$$
R^{2}(t)=\frac{1}{16} \frac{x_{0}}{Q_{s}\left(t_{0}\right)} \exp \left\{\frac{\alpha_{s} N_{c}}{2 \pi} \epsilon t\right\}
$$

with

$$
\frac{\alpha_{s} N_{c}}{2 \pi} \epsilon=\frac{a^{2}}{2}\left[-1+\sqrt{1+4 \frac{\omega}{a^{2}}}\right] .
$$

Numerically we find

$$
\frac{\alpha_{s} N_{c}}{2 \pi} \epsilon=.87 \omega .
$$

Thus, as claimed we arrive at the exponential growth of the total cross section.

The exact value of $\epsilon$ given in Eqs. (22),(23) should not be taken too seriously. The point is that the explicit form of the dipole density Eq. (20) was derived by a saddle point integration, and as such is valid only for $\ln \left(16 b^{2} / x_{0} x\right)<\alpha_{s} t$. Since this condition is not satisfied by Eq. (21), we cannot strictly speaking use the saddle point expression Eq. (20). This ambiguity however affects only the numerical value of $\epsilon$ and not the parametric dependence in Eq. (21). The reason is that even beyond the saddle point approximation the density has the form

$$
n\left(x_{0}, x, b, t\right) \propto \frac{1}{x^{2}} \exp \left\{\alpha_{s} t F\left(\frac{\ln \frac{16 b^{2}}{x_{0} x}}{\alpha_{s} t}\right)\right\}
$$

The relevant condition is $F=0$, and thus the solution parametrically must be the same as Eq. (21).

It is important to realize, that although we use the BFKL dipole density of Eq. (20), our argument does not assume that the scattering probability at $R(t)$ is dominated by one Pomeron exchange. The only assumption is that parametrically the total unitarized probability is the same as the one Pomeron one. We use the criterion of dipole density only as an indicator for the magnitude of the total probability. The total scattering probability is given by 


$$
P(b)=\sum_{m=1}^{\infty} \gamma_{m}(x, r) P_{m}\left(x_{0}, x, b, t\right),
$$

where $P_{m}\left(x_{0}, x, b, t\right)$ is the probability to find in the projectile wave function $m$ dipoles of size $x$ at transverse coordinate $b$ within the area of the target radius $R_{0}$ and $\gamma_{m}$ is the probability of the scattering of an $m$-dipole state. In fact one should also sum over all dipole sizes smaller than $R_{0}$. We have neglected this summation in Eq. (25) thus somewhat underestimating the total probability. Since $\gamma_{m} \geqslant \gamma$, the probability is bounded from below as

$$
P(b) \geqslant \gamma \sum_{m=1}^{\infty} P_{m}\left(x_{0}, x, b, t\right) .
$$

For dipoles of size $Q_{s}^{-1}$, the scattering probability $\gamma$ is of order unity. Thus the only condition that we use is

$$
\sum_{m=1}^{\infty} P_{m}\left(x_{0}, x, b, t\right)=O(1)
$$

whenever

$$
n\left(x_{0}, x, b, t\right)=\sum_{m=1}^{\infty} m P_{m}\left(x_{0}, x, b, t\right)=O(1) .
$$

The only way this condition can be violated, is if the wave function is dominated (with exponential accuracy) by the trivial configuration with no dipoles, even when the average dipole number is one. Although the dipole model wave function is known to have relatively large fluctuations, there is nothing in its known properties [7,11] to suggest such an extreme behavior. In fact for the explicit exponential model used in $[7,11]$ our condition clearly holds.

To summarize, in the target rest frame, the violation of unitarity by the BK evolution can be understood as follows: Start with a single dipole scattering on the hadronic target of transverse size $R_{0}$. With increasing energy the projectile dipole emits additional dipoles strictly according to the BFKL evolution. The density as well as the transverse size of the projectile state thus grows. The increase in density leads to increasing importance of multiple scatterings which are properly accounted for in the BK derivation. This ensures that the scattering probability saturates locally. In the saturation regime, as long as the size of the projectile state $R(t)$ is smaller than the target size $R_{0}$, the cross section grows essentially only due to surface effects,

$$
\sigma=\pi R_{0}^{2}+2 \pi R_{0} x_{0} \exp \left[\frac{\alpha_{s} N_{c}}{2 \pi} \epsilon t\right] .
$$

As long as $\left(\alpha_{s} N_{c} / 2 \pi\right) \epsilon t<\ln \left(R_{0} / x_{0}\right)$, the cross section is practically geometrical. However once the energy is high enough, so that the projectile size is larger than that of the target, the total cross section is determined by the former and grows exponentially with rapidity according to Eq. (21). Thus at rapidities

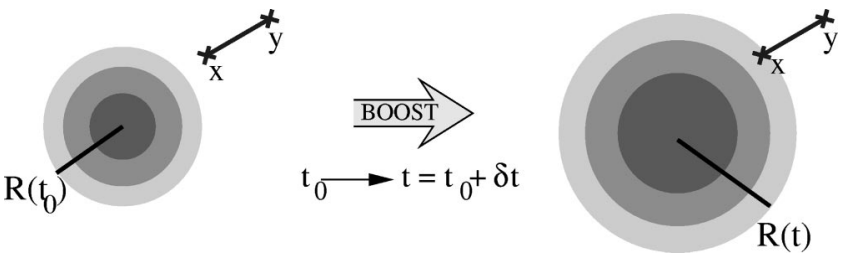

FIG. 4. The BK evolution in the target evolution picture. As long as the dipole leg $y$ is in the white region, only the evolution of the scattering amplitude $U(x)$ in Eq. (33) is nontrivial.

$$
t>\frac{1}{\alpha_{s} N_{c}} \ln \frac{R_{0}}{x_{0}}
$$

the BK evolution is nonunitary and cannot be applied.

This also illustrates that the applicability of the BK evolution crucially depends on the nature of the target. If the target is thick enough, so that the multiple scatterings become important before the growth of the projectile radius does, and if the target is wide enough, so that saturation occurs before the projectile radius swells beyond that of the target, then there is an intermediate regime in which the inelastic cross section remains practically constant and equal to $\pi R_{0}^{2}$. Then $\mathrm{BK}$ applies in this intermediate regime. However, if the target is a nucleon, neither one of these conditions is satisfied. Thus the tainted infrared behavior of the BFKL evolution of the projectile will show up right away and will invalidate the application of the BK equation.

\section{THE TARGET EVOLUTION PICTURE}

The BK equation (9) is valid only in the leading approximation in $1 / N_{c}$. Beyond the leading order the evolution for a dipole cross section does not close, but rather is the first in the infinite hierarchy of equations. This hierarchy was derived in [5]. It is useful to consider its interpretation in the frame where all the energy resides in the target. In this frame further increase in energy leads to growth of the target gluon fields. The evolution equation governs the change in the distribution of the gluon fields $A_{\mu}$ in the wave function of the target. In the particular gauge used in [5], the largest component of the vector potential is $A^{+}$. In this gauge the S-matrix for scattering of a fast fundamental projectile on the target fields is given by the unitary eikonal factor $U(x)$

$$
U(x)=P \exp \left\{i \int d x^{-} T^{a} A_{a}^{+}(x)\right\},
$$

where $T^{a}$ are the generators of the $S U(N)$ group in the fundamental representation. The first in the hierarchy of evolution equations derived in [5] is

$$
\begin{aligned}
\frac{d}{d t} \operatorname{Tr}\left\langle 1-U^{\dagger}(x) U(y)\right\rangle= & \frac{\alpha_{s}}{2 \pi^{2}} \int d^{2} z \frac{(x-y)^{2}}{(x-z)^{2}(y-z)^{2}} \\
& \times\left\langle N_{c} \operatorname{Tr}\left[U^{\dagger}(x) U(y)\right]\right. \\
& \left.-\operatorname{Tr}\left[U^{\dagger}(x) U(z)\right] \operatorname{Tr}\left[U^{\dagger}(z) U(y)\right]\right\rangle .
\end{aligned}
$$


The averaging in Eq. (32) is taken over the ensemble of field strengths characterizing the target, i.e., over the target wave function. In the large $N_{c}$ limit the averages in Eq. (32) factorize and one recovers Eq. (9) with the identification (see Fig. 4)

$$
N(x, y)=\frac{1}{N_{c}} \operatorname{Tr}\left\langle 1-U^{\dagger}(x) U(y)\right\rangle .
$$

A physically appealing reformulation of Balitsky's hierarchy of equations was given by Weigert [25] in terms of a nonlinear stochastic process. The unitary scattering amplitude $U$ evolves under the action of a stochastic source

$$
\begin{aligned}
\frac{d U(x)}{d t}= & g U(x) i T^{a} \int \frac{d^{2} z}{\sqrt{4 \pi^{3}}} \frac{(x-z)_{i}}{(x-z)^{2}}[1 \\
& \left.-\widetilde{U}^{\dagger}(x) \widetilde{U}(z)\right]^{a b} \xi_{i}^{b}(z) \\
& +\frac{i \alpha_{s}}{2 \pi^{2}} U(x) T^{a} \int d^{2} z \frac{1}{(x-z)^{2}} \operatorname{Tr}\left[T^{a} \widetilde{U}^{\dagger}(x) \widetilde{U}(z)\right],
\end{aligned}
$$

where $U(x)$ and $\widetilde{U}(x)$ are the unitary matrices (31) in the fundamental and adjoint representations, respectively. The white noise $\xi$ is characterized by Gaussian local correlations

$$
\left\langle\xi_{i}^{a}\left(t^{\prime}, z^{\prime}\right) \xi_{j}^{b}\left(t^{\prime \prime}, z^{\prime \prime}\right)\right\rangle=\delta^{a b} \delta_{i j} \delta\left(t^{\prime}-t^{\prime \prime}\right) \delta\left(z^{\prime}-z^{\prime \prime}\right) .
$$

This Langevin equation gives rise to an infinite number of equations for correlators of $U$ which coincide with those derived in [5].

Consider then the Langevin equation formulation, Eq. (34). Assume that at the initial rapidity $t_{0}$ the target is black within radius $R_{0}$. This means that for $|z|<R_{0}$ the matrix $U(z)$ fluctuates very strongly so that it covers the whole group space. Let us concentrate on the point $x$ which is initially outside of this black region. The matrix $U(x)$ then is close to unity. Thus there is no correlation between $U(x)$ and $U(z)$, and the second and third terms on the right hand side of Eq. (34) can be set to zero. This is the random phase approximation introduced in [25] and used later in [19]. Note that this approximation does not linearize the evolution. Rather it corresponds to equating the nonlinear term in Eq. (9) to unity for $z$ in the black region.

As the target field ensemble evolves in rapidity, the radius of the black region grows. As long as the point $x$ stays outside the black region, we can approximate the Langevin equation by

$$
\frac{d}{d t} U(x)=-\sqrt{\frac{\alpha_{s} N_{c}}{2 \pi^{2}}} \int_{|z|<R} d^{2} z \frac{(x-z)_{i}}{(x-z)^{2}} \xi_{i}(z) .
$$

Here we did not indicate explicitly color indices, since they are inessential to the argument. We have also neglected the contribution to the derivative of $U$ that comes from gluons originating from the sources outside the black region. Those contributions speed up the growth of $U$, and so by omitting them we can only underestimate the rate of growth of the radius of the black region. The formal solution of Eq. (36) is

$$
1-U(x, t)=\sqrt{\frac{\alpha_{s} N_{c}}{2 \pi^{2}}} \int_{t_{0}}^{t} d \tau \int_{|z|<R(\tau)} d^{2} z \frac{(x-z)_{i}}{(x-z)^{2}} \xi_{i}(z) .
$$

Squaring it and averaging over the noise term gives

$$
\left\langle[1-U(x, t)]^{2}\right\rangle=\frac{\alpha_{s} N_{c}}{2 \pi^{2}} \int_{t_{0}}^{t} d \tau \int_{|z|<R(\tau)} d^{2} z \frac{1}{(x-z)^{2}} .
$$

As long as $x$ is outside the black region and $|x|>R$, we can approximate the integral on the right hand side by

$$
\int_{|z|<R(\tau)} d^{2} z \frac{1}{(x-z)^{2}}=\pi \frac{R^{2}(\tau)}{x^{2}},
$$

and Eq. (38) becomes

$$
\left\langle[1-U(x, t)]^{2}\right\rangle=\frac{\alpha_{s} N_{c}}{2 \pi} \frac{1}{x^{2}} \int_{t_{0}}^{t} d \tau R^{2}(\tau) .
$$

As the black region grows, eventually it will reach the point $x$. At this rapidity the matrix $U(x)$ starts fluctuating with an amplitude of order 1 . Thus, when $R(t)=|x|$, the left hand side of Eq. (40) becomes a number of order 1, which we call $1 / \epsilon$. We thus have an approximate equation for $R(t)$,

$$
\frac{1}{\epsilon} R^{2}(t)=\frac{\alpha_{s} N_{c}}{2 \pi} \int_{t_{0}}^{t} d \tau R^{2}(\tau),
$$

or in the differential form

$$
\frac{d}{d t} R(t)=\frac{\alpha_{s} N_{c}}{4 \pi} \epsilon R(t) .
$$

At large rapidities therefore the radius of the black region is exponentially large

$$
R(t)=R\left(t_{0}\right) \exp \left\{\frac{\alpha_{s} N_{c}}{4 \pi} \epsilon\left(t-t_{0}\right)\right\} .
$$

We thus recover the result of the previous section.

The approximations leading to Eq. (42) are not strictly speaking valid when the point $x$ is on the boundary of the black region. First, Eq. (39) is an underestimate of the integral, since the inequality $|x| \gg R$ no longer holds. However, this approximation can lead only to an underestimate of the rate of growth of $R$. Second, not for all points $z$ in the black region the term $U(x) U^{\dagger}(z)$ in Eq. (34) can be dropped. This however is also unimportant, since when $x$ is on the boundary of the black region, although the factor [1 $\left.-U(x) U^{\dagger}(z)\right]$ is not strictly unity, it is still of order 1 for all points $z$. It is in fact different from unity only for points $z$ in the vicinity of $x$. Thus, although we cannot determine the exact numerical value of $\epsilon$, the functional form of the solution as well as its parametric dependence is given correctly by Eq. (43).

From the point of view of the evolution of target fields the violation of the unitarity can be interpreted as follows. The 
RHS of Eq. (34) is nothing but the total Coulomb (Weizsäcker-Williams) field at point $x$ due to the color charge sources at points $z$. The stochastic noise tells us that these color sources are uncorrelated both in the transverse plane and in rapidity. For such random sources the square of the total color charge is proportional to the area, and this is precisely the factor $R^{2}$ in Eq. (40). The incoming dipole thus scatters on the Coulomb field created by a large incoherent color charge. Because the Coulomb field is long range, the whole bulk of the region populated by the sources contributes to the evolution and leads to rapid growth of $R$. If the field created by the sources was screened by some mass, the evolution would be perfectly unitary. To illustrate this point, let us substitute the Coulomb field $(x-z)_{i} /(x-z)^{2}$ in Eq. (34) by an exponentially decaying field $m \exp \{-m|x-z|\}$. It is straightforward to perform now the same analysis as before. Equation (39) is now replaced by

$$
\int_{|z|<R(\tau)} d^{2} z m^{2} \exp \{-m|x-z|\}=\exp \{-m|x-R|\} .
$$

This leads to the substitution $R^{2} \rightarrow \exp \{m R\}$ in all subsequent equations with the end result that

$$
R(t)=\alpha_{s} \frac{\epsilon}{m} t
$$

which in fact saturates the Froissart bound.

Thus the reason for the violation of unitarity is that the evolution is driven by the emission of the long range Coulomb field from a large number of incoherent color sources in the target.

To conclude this section we would like to discuss the relation of our results with numerical studies of the nonlinear QCD evolution. Studies within the framework of the dipole model were reported in [11]. Reference [11] does not deal directly with the nonlinear BK equations, but rather with the onium-onium scattering in the framework of the dipole model. However as is clear from our discussion at asymptotically high energies this distinction is irrelevant. The growth of the transverse size of the projectile eventually determines the behavior of the cross section irrespective of the nature of the target. Indeed our results are in agreement with those of [11]. The numerical results of [11] clearly indicate that even though the scattering probability is unitarized locally in the impact parameter space, the total cross section keeps on growing exponentially with $t$ (Figs. 9 and 10 of [11]). From Fig. 10 of [11] we conclude that the power of the exponential is about $.75 \omega$, where $\omega$ is the leading BFKL exponential. Interestingly, our rough estimate (23) is in reasonable qualitative agreement with this numerical result.

Our results Eqs. (21),(42) are in apparent contradiction with the conclusions of numerical work $[15,16]$. The origin of this discrepancy is that these references solve Eq. (9) within the local approximation, assuming that important contributions come only from the dipole sizes which are smaller than the impact parameter. Within this approximation the de- pendence on the impact parameter in Eq. (9) becomes parametric, and the growth of the total cross section is determined entirely by the shape of the initial condition. The Froissart bound is then saturated for the exponential initial profile of $N(b)$. The physics here is simple. In the local approximation of $[15,16]$ the gluon density of the target (and the scattering probability $N$ ) evolves at all impact parameters $b$ according to the same exact translationally invariant equation. The density locally grows at all impact parameters at the same rate. This rate is the same as the growth of the saturation momentum and is powerlike with rapidity, Eq. (13). Thus if one starts from an initial configuration with the exponential density profile $g(b)=\exp \left\{-b / R_{0}\right\}$, after the evolution to rapidity $t$ it becomes

$$
g(b, t)=\exp \left\{-b / R_{0}+\alpha_{s} \lambda t\right\}
$$

The scattering probability on such a system is unity at impact parameters for which $g(b) \geqslant 1$. Thus the highest impact parameter that contributes to the total cross section at rapidity $t$ is $b_{\text {max }}=\left(\alpha_{s} \lambda / R_{0}\right) t$, and the cross section ${ }^{5}$ is $\sigma=\pi b_{\text {max }}^{2}$ $\propto t^{2}$. For initial Gaussian distribution on the other hand, the same argument leads to the linear growth of the cross section with $t$.

This feature, namely that the asymptotic form of $\sigma$ is determined by the initial distribution, is clearly an artifact of the local approximation. The reason the local approximation leads to this behavior is that it neglects the effects of far away black regions (where $N=1$ ) on the scattering probability in the gray areas (where $N<1$ ). As is apparent from our analysis in Eqs. (36)-(40), it is precisely the effect of the far away black regions that drives the growth of the total cross section. This is due to the long range Coulomb fields originating in the central black region. In fact, the only contributions we kept on the RHS of Eq. (36) are due to dipoles with sizes of the order of the impact parameter. In this respect our discussion is orthogonal to that of $[15,16]$. It is clear from the comparison of our results to those of $[15,16]$ that the effect of these long range fields on the total cross section is far greater than that of the local translationally invariant part of the evolution. Even if one starts from an exponential density profile, the full BK evolution generates powerlike and not exponential tails in the density at large $t$. These powerlike tails dominate the total cross section and lead to the universal exponential growth of $\sigma$ with rapidity. The local approximation is adequate for studying the behavior of $Q_{s}(t)$ in the dense central region, as this is determined by local effects. It is however not a good approximation for the total cross section, which is dominated by the evolution of long range Coulomb fields.

\footnotetext{
${ }^{5}$ In this discussion we neglect the dependence of $N$ on the size of the dipole. Strictly speaking such dependence is of course present, and it determines the rapidity at which the asymptotic behavior of $\sigma$ sets in. The asymptotic form of $\sigma$ however is independent of the dipole size.
} 


\section{ONE POMERON, TWO POMERON; HARD POMERON, SOFT POMERON}

The linear evolution of the projectile wave function inherent in the BK evolution is a direct consequence of the large $N_{c}$ limit. In this limit one can neglect the interactions between the dipoles in the projectile wave function. Individual Feynman diagrams which contain dipole-dipole interactions are suppressed by powers of $1 / N_{c}$. Thus, the dipoles in the projectile wave function do not interact during the evolution and they scatter independently of each other. However, the number of interacting diagrams grows very fast with the number of interacting dipoles. As the number of dipoles in the wave function which can interact with each other becomes $O(N)$, the number of the suppressed diagrams becomes $O\left(N^{2}\right)$ and the suppression disappears. At high enough rapidity, where the dipole-dipole interactions are important, the evolution equation (9) breaks down and wave function saturation effects start to play an essential role. Although Eq. (32) contains some $1 / N_{c}$ corrections, those are only "group theoretical" corrections reflecting the fact that at finite $N_{c}$ a gluon is not strictly equivalent to a $\bar{q} q$ pair. It does not contain the corrections due to the interactions of gluons (or dipoles) in the projectile wave function.

The interaction probability of a dipole of size $r$ with another dipole of similar size in the projectile wave function is of the order $\alpha_{s} r^{2} n(r)$, while the probability of the direct interaction with the target is $\gamma(r)$. The multiple scattering corrections are thus more important as long as $\alpha_{s} r^{2} n(r)$ $\ll \gamma(r)$. The projectile wave function corrections become important as the density grows so that

$$
r^{2} n(r)=\exp \{\omega t\}=\frac{\gamma}{\alpha_{s}}
$$

Assuming that for a large target $\gamma$ is of order 1 , this happens for rapidities

$$
t \propto \frac{1}{\omega} \ln 1 / \alpha_{s} \sim \frac{1}{\left(N_{c} \alpha_{s}\right)} \ln 1 / \alpha_{s} .
$$

The "subleading" large $N_{c}$ nature of these corrections is clear if one traces back the explicit $N_{c}$ dependence of Eq. (48). In fact, the coupling constant $\alpha_{s}$ occurs in $n(r)$ only in the combination $\alpha_{s} N_{c}$, since the BFKL evolution neglects dipole-dipole interactions. Thus the $\alpha_{s}$ in the numerator of Eq. (47) is explicitly subleading in $N_{c}$ and indeed accounts for dipole-dipole interactions.

The above illustrates that the BK resummation improves on the BFKL evolution when the target is dense, that is the scattering probability $\gamma$ is larger than order $\alpha_{s}$. On a large nucleus of atomic number $A$ one expects $\gamma \propto \alpha_{s} A^{1 / 3}$, and thus in this case $\mathrm{BK}$ resums all corrections in powers of $\alpha_{s} A^{1 / 3}$ [6]. However even in this case the validity of the BK equation remains limited. The scattering probability $\gamma$ is only large at central impact parameters. At peripheral impact parameters the density in the target drops to zero, and so for the peripheral scattering events the applicability of $\mathrm{BK}$ is no better than that of BFKL. In particular, the diffusion contri- butions play the same role, since they are not associated with high partonic densities. Since at very high energies the total cross section is dominated by peripheral events, one does not expect the BK evolution to be a valid approximation in calculating the total cross section. Comparing Eqs. (30) and (48) we conclude that for large targets, at rapidities at which the total cross section is dominated by peripheral impact parameters, the wave function saturation effects are important even for small projectiles. Thus even within perturbation theory, the wave function saturation effects are bound to be the ones that determine the growth of the total cross section with energy.

Could wave function saturation effects lead to a perturbative unitarization of the total cross section? As seen above, the violation of the Froissart bound in the BK approximation is due to large and incoherent color charge fluctuations in the black region. If there was a mechanism to ensure strong correlations such that the total color charge in a region of fixed size $L$ is zero, then the incoming dipole would feel the Coulomb field only within the fixed distance $L$ from the black region. Thus the new charges produced by the evolution would only "split off" the edges of the black region rather than from its bulk. This scenario is equivalent to exponential decay of the field, and would lead to a unitary evolution, as expected for a confining theory like QCD. However, it does not seem plausible that this type of correlation can be enforced on the system by a perturbative mechanism, nor that perturbation theory can generate any kind of "mass" for the gluons which would lead to exponential decay of gluon fields generated by sources in the black region. We thus expect that any perturbative corrections will generate powerlike tails of gluon density at large distances from the black region. The growth of these power tails with rapidity will inevitably lead to an exponential growth of the cross section.

We expect, however, that wave function saturation effects diminish the exponent $\epsilon$ significantly relative to the BK value $\left(\alpha_{s} N_{c} / 2 \pi\right) \epsilon=.75 \omega$. The evolution equations derived in [4] go beyond the BK equation by including wave function saturation effects. They provide a well-defined perturbative framework for calculating this effect.

We have seen in the previous sections that QCD perturbation theory predicts the existence of two distinct physical mechanisms for the power growth of hadronic cross sections with energy. One is due to the fast growth of the partonic densities, while the other is due to expansion of hadronic states in the transverse plane. The first mechanism is the leading one for systems containing a small number of partons. When the partonic density reaches the critical value of order $1 / \alpha_{s}$, further growth of the density is cut off by the perturbative saturation effects. The transverse expansion mechanism is however likely to survive in this situation, and thus it should become the leading driving force for the perturbative growth of cross sections in dense systems.

The first mechanism with the perturbative BFKL exponent of around $.3-.4$ fits very nicely with the so called hard Pomeron utilized to fit the hadronic cross sections of small systems [26]. The exponent due to the transverse expansion has not been calculated yet, although the equations which determine it have been derived in [4]. Although we do not 
know yet the value of this exponent, we know for certain, that it is smaller than the BFKL one. One is naturally led to ask whether this perturbative growth of the transverse size of saturated systems underlies the experimentally observed power growth of the cross sections in purely hadronic processes, like $p p$ and $\bar{p} p$. This type of expansion, and with it the powerlike growth of the cross section, should cease at asymptotically high energies, where the perturbation theory must become invalid.

This picture suggests that nonperturbative effects are called for only in order to unitarize the cross section at asymptotically high energies, but not in order to furnish the mechanism for its fast growth in the pre-asymptotic regime. This is also natural from the following perspective. The Froissart-like behavior is associated with the existence of a gap in the spectrum, as can be illustrated by a simple and intuitive argument due to Heisenberg [14]. In a theory with a mass gap, the profile of the distribution of matter density in any target must decay exponentially at the periphery, $\rho(b)$ $\propto \exp \{-m b\}$. As this target is struck by a projectile, in order to produce an inelastic scattering event at least one particle must be produced. Assuming that the scattering is local in the impact parameter plane, the region of the overlap of the probe and the target must therefore contain energy at least equal to the mass of the lightest particle, $m$. For scattering at energy $E=s / m$ in the frame where all the energy resides in the target, the target energy density is $E \rho(b)$. Thus the scattering can only take place for impact parameters smaller than those that satisfy $E \exp \{-m b\}=m$. Thus $b_{\max }$ $=(1 / m) \ln \left\{s / m^{2}\right\}$, which is equivalent to the Froissart bound. Conversely, if the cross section grows as a power of energy, then the density distribution in the target is not exponential but powerlike. With $\rho(b) \propto b^{-\lambda}$ one obtains $b_{\max } \propto s^{1 / \lambda}$. Since the power growth of hadronic cross sections persists in a large interval of energies, one expects that for a range of impact parameters the density distribution in hadronic states is powerlike. Perturbation theory provides a natural explanation of such a powerlike distribution. The tails of perturbative distribution are due to massless gluon fields emitted from the color charges in the target. Even though the target is neutral, it always possesses a multipole moment of some order, and thus perturbatively is always accompanied by a long range powerlike tail of a massless gluon field. The precondition for the applicability of this perturbative mechanism in hadronic systems is that hadrons themselves are built from small "black" building blocks. As explained earlier, the gluon fields at the periphery are emitted from the bulk of the black disk, and not from its boundary. Thus, in order to explain the preasymptotic powerlike rise of hadronic cross sections by perturbative Coulomb-like gluon fields, the radius of these black region must be smaller than the confining scale of QCD. It is in fact widely believed that QCD does naturally contain the scale of the right order-the scale associated with chiral symmetry breaking. In particular the radius of a constituent quark is believed to be at most $.3 \mathrm{fm}$ and perhaps even smaller [27]. This supports the phenomenological picture of the proton as a loosely bound system of three

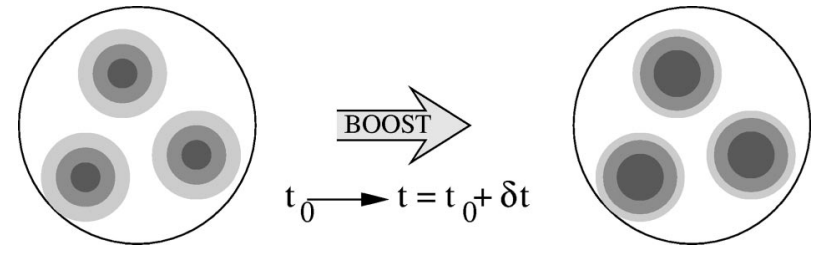

FIG. 5. Schematic picture of the proton as a loosely bound system of three constituent quarks which provide "black" building blocks at initial rapidity $t_{0}$. Under boost, the blackness of these regions does not change since the gluon density is saturated, but the transverse size of the black region grows.

small constituent quarks. The nonperturbative confining force keeps these quarks confined within the spatial region of radius $.8-.9 \mathrm{fm}$.

A quantitative analysis of the nonlinear evolution equations [4] which could substantiate our proposal of the perturbative soft Pomeron, is not available yet. There are however some qualitative consequences of the above picture that can be compared to experimental data. Let us check first whether the value of the cross section in our model is in rough agreement with experiment. The total cross section of $p p$ scattering at the lowest energy where the Reggeon contributions are not important, $\sqrt{s} \propto 50 \mathrm{GeV}$, is $45 \mathrm{mb}$. The total cross section for the scattering of two black disks of diameter $d$ is $2 \pi d^{2}$. Thus in the simplest model in which the proton is composed of three completely "black" constituent quarks of diameter $d$, the total $p p$ cross section is $3 \times 3 \times 2 \pi d^{2}$. Equating this to $45 \mathrm{mb}$ we find $d=.28 \mathrm{fm}$. This should be considered as a lower bound on the value of $d$. It is more likely that the constituent quarks are only black in the center and have gray peripheral regions, see Fig. 5. For peripheral $q q$ events the scattering occurs with probability $f<1$. Incorporating this roughly as an average "grayness" factor in the formula for the cross section gives $d=.28 f^{-1 / 2} \mathrm{fm}$. For $f=.5$ we have $d=.4 \mathrm{fm}$. We may thus think of the proton as a collection of three loosely bound constituent quarks each described by a disk of $d=.3-.4 \mathrm{fm}$ which is essentially black in its center but gray at its boundary, see Fig. 5 .

This picture is quite remarkable, since the "active" area inside the proton in our model is much smaller than the proton radius and thus one may have worried that the model will underestimate the total cross section. This however does not happen. Further support for this picture comes from the ratio $\sigma_{\pi p} / \sigma p p$ which is very close to $2 / 3$. The Pomeron contributions to the total hadronic cross sections are parametrized as $\sigma_{p p}=21.70 s^{0.808}$ and $\sigma_{\pi p}=13.63 s^{0.808}$ [28]. This is consistent with $\pi$ having two constituent quarks.

Another feature of the model is that although the quarks are black, the proton itself is not. Thus we expect the ratio of the elastic to total cross section to be well below the black disk value of $1 / 2$. The experimental value of this ratio at $\sqrt{s}=50 \mathrm{GeV}$ is indeed just below $1 / 5$ [28].

Another global characteristic of the scattering is the radius of the proton as measured via the shrinkage of the elastic peak, $d \sigma_{e l} / d t \propto \exp \left\{\left(R^{2} / 4\right) t\right\}$. For the elastic $p p$ scattering this gives the value of $R$ consistent with the proton radius $R=.8 \mathrm{fm}$ [28]. Again at first sight this sounds like trouble, 
since it is much larger than the radius of a constituent quark. However one should realize that the radius of the individual quark is not relevant for this particular quantity. The process is elastic if the proton as a whole emerges from the interaction intact. Thus indeed it is the radius of the proton and not of a quark that should determine the $t$-dependence of the elastic cross section in our model. The radius of the quark may emerge in a similar way in the processes "elastic" with respect to individual constituent quark scattering. Whether one can define a subset of final states that correspond to such a process is an interesting question, but at present it is not clear to us how to do it.

The picture of the proton as built from three small constituent quarks was invoked in the nonperturbative model for the high energy scattering in [12]. The physics of [12] is however quite different from that of our proposal. The quarks themselves in [12] are not thought of as being black, and the growth of the quark-quark cross section is due to the increase of the density of the gluon cloud surrounding an individual quark, rather than to the increase in its transverse size. Reference [13] also appeals to the scale of .3 fermi although not explicitly in connection with the size of constituent quarks. Again, however the mechanism of the growth of the cross section in [13] appears to be the same as the leading BFKL mechanism, that is the growth of gluonic density. The role of instanton effects in [13] is to limit the gluon emissions only to within the transverse sizes smaller than .3 Fermi, and thus to cut off the expansion in the transverse plane. In contrast our picture assumes that nonperturbative effects (perhaps instantons) are responsible for the buildup of a black gluon cloud around each quark at low energy. The subsequent evolution in energy is dominated by perturbative swelling of these black regions. Eventually, when the size of the quarks reaches confining scale, other nonperturbative effects kick in and cut off further powerlike growth of the cross section. The physics of these nonperturbative effects is presumably the physics of confinement.

We note also that the two distinct physical mechanisms for two Pomerons have a consequence that they will appear with different probability in different processes. For example in $p p$ scattering there are rare proton configurations which do not have the typical hadronic structure, but contain quarks closely bunched together in coordinate space. Those are the configurations responsible for the color transparency effects in the hadronic scattering [29]. These configurations are rare and short lived. Thus they do not have time to develop the gluon clouds around the quarks that could make them "black." These configurations will predominantly evolve towards the increase in density, and thus will have the energy dependence of the hard Pomeron. This is consistent with the findings of [26] that a hard Pomeron is present already in purely hadronic processes.

On the other hand one does not necessarily expect the soft Pomeron to appear in DIS even at low $Q^{2}$. The reason is that even though at low $Q^{2}$ the (DIS) cross section has large contributions from the photon fluctuations into the states of hadronic size, these states do not live long enough on the hadronic time scale, and thus do not have time to develop dense gluon clouds around the quarks. The energy dependence of such large but dilute states would then be of the hard Pomeron nature. This appears to be consistent with the recent results which do not require the soft Pomeron to fit DIS data even at very small $Q^{2}[30]$.

Perhaps the most appealing feature of this scenario is that it gives hope to understand the soft Pomeron within the well defined, bona fide perturbative framework. The equations that resum the wave function saturation effects have been derived in [4]. Even though their numerical study is probably much more involved than that of Eqs. (9),(32), we think the question is interesting enough to motivate such an undertaking.

\section{ACKNOWLEDGMENTS}

This work has been supported in part by PPARC. U.A.W. thanks the Department of Mathematics and Statistics, University of Plymouth for hospitality while part of this work was being done. We are indebted to J.G. Milhano and H. Weigert for interesting discussions during the early stages of this work. We also thank M. Braun, Yu. Kovchegov, E. Levin and K. Tuchin for useful discussions and correspondence.
[1] E.A. Kuraev, L.N. Lipatov, and V.S. Fadin, Sov. Phys. JETP 45, 199 (1977); Ya.Ya. Balitskiı̌ and L.N. Lipatov, Sov. J. Nucl. Phys. 28, 21 (1978).

[2] L.V. Gribov, E. Levin, and M. Ryskin, Phys. Rep. 100, 1 (1983); A. Mueller and J.W. Qiu, Nucl. Phys. B286, 427 (1986).

[3] L. McLerran and R. Venugopalan, Phys. Rev. D 49, 2233 (1994); 49, 3352 (1994).

[4] J. Jalilian Marian, A. Kovner, A. Leonidov, and H. Weigert, Nucl. Phys. B504, 415 (1997); Phys. Rev. D 59, 014014 (1999); 59, 034007 (1999); 59, 099903 (1999); J. Jalilian Marian, A. Kovner, and H. Weigert, ibid. 59, 014015 (1999); A. Kovner and J.G. Milhano, ibid. 61, 014012 (2000).

[5] I. Balitskiı̌, Nucl. Phys. B463, 99 (1996); Phys. Rev. Lett. 81, 2024 (1998); Phys. Rev. D 60, 014020 (1999).
[6] Yu. Kovchegov, Phys. Rev. D 60, 034008 (1999).

[7] A. Mueller, Nucl. Phys. B415, 373 (1994); B437, 107 (1995).

[8] A. Kovner, J.G. Milhano, and H. Weigert, Phys. Rev. D 62, 114005 (2000).

[9] E. Ferreiro, E. Iancu, A. Leonidov, and L. McLerran, Nucl. Phys. A703, 489 (2002).

[10] A. Kovner and U.A. Wiedemann, Phys. Rev. D (to be published), hep-ph/0112140.

[11] G.P. Salam, Nucl. Phys. B461, 512 (1996).

[12] B.Z. Kopeliovich, I.K. Potashnikova, B. Povh, and E. Predazzi, Phys. Rev. D 63, 054001 (2001).

[13] D. Kharzeev and E. Levin, Nucl. Phys. B578, 351 (2000); D.E. Kharzeev, Y.V. Kovchegov, and E. Levin, Nucl. Phys. A690, 621 (2001).

[14] W. Heisenberg, Z. Phys. 133, 65 (1952) as quoted in A. Hebecker, hep-ph/0111092. 
[15] E. Levin and K. Tuchin, Nucl. Phys. B573, 833 (2000); Nucl. Phys. A693, 787 (2001).

[16] M.A. Braun, Eur. Phys. J. C 16, 337 (2000).

[17] K. Golec-Biernat, L. Motyka, and A.M. Stasto, Phys. Rev. D 65, 074037 (2002); E. Levin and M. Lublinsky, Nucl. Phys. A696, 833 (2001)

[18] Yu. Kovchegov, Phys. Rev. D 61, 074018 (2000).

[19] E. Iancu and L. McLerran, Phys. Lett. B 510, 145 (2001); E. Iancu, K. Itakura, and L. McLerran, Nucl. Phys. A708, 327 (2002).

[20] K. Golec-Biernat and M. Wustoff, Phys. Rev. D 60, 114023 (1999).

[21] A. Mueller, Nucl. Phys. B558, 285 (1999).

[22] E. Gotsman, E.M. Levin, and U. Maor, Eur. Phys. J. C 5, 303 (1998).
[23] A. Kovner and U.A. Wiedemann, Phys. Rev. D 64, 114002 (2001).

[24] J.R. Forshaw and D.A. Ross, Quantum Chromodynamics and the Pomeron (Cambridge University Press, Cambridge, England, 1997).

[25] H. Weigert, Nucl. Phys. A703, 823 (2002).

[26] A. Donnachie and P.V. Landshoff, Phys. Lett. B 437, 408 (1998); 518, 63 (2001); A. Donnachie and H.G. Dosch, Phys. Rev. D 65, 014019 (2002).

[27] S. Bondarenko, E. Levin, and J. Nyiri, hep-ph/0204156.

[28] E.A. De Wolf, J. Phys. G 28, 1023 (2002).

[29] L. Frankfurt, V. Guzey, and M. Strikman, J. Phys. G 27, R23 (2001).

[30] A. Donnachie and P.V. Landshoff, hep-ph/0204165. 